\title{
Algorithms and Protocols in Information and Communication Systems
}

\author{
Wail Mardini and Yaser Khamayseh \\ Department of Computer Science, \\ Jordan University of Science and Technology, Irbid (Jordan) \\ Tel: +962- 7201000 E-mail: mardini@just.edu.jo, yaser@just.edu.jo
}

Received: September 6, 2012 Accepted: September 25, 2012 Published: September 29, 2012

DOI: $10.5296 /$ npa.v4i3.2469

URL: http://dx.doi.org/10.5296/npa.v4i3. 2469

\begin{abstract}
This special issue includes extended versions of the best papers presented in the Third International Conference on Information and Communication Systems (ICICS 2012) and some invited research paper for distinguished participants in this conference. The conference took place in Irbid, Jordan, during the period of April 3-5, 2012.
\end{abstract}

Keywords: Message Routing Protocols, Wireless Body Area Networks, Complex SSC/MRC combiner, Intrusion detection, Backoff algorithm, Fibonacci, IEEE 802.15.4.

\section{Introduction}

The International Conference on Information and Communication Systems (ICICS 2012) is a forum for scientists, engineers, and practitioners to present their latest research results, ideas, developments, and applications in all areas of Computer and Information Sciences. Many topics were covered including Artificial Intelligence, Mobile Computing, Networking, Information Security and Cryptography, Intrusion Detection and Computer Forensics, Web Content Mining, Bioinformatics and IT Applications, Database Technology, Systems Integration, Information Systems Analysis and Specification, Telecommunications, and Human-Computer Interaction.

This special issue includes five papers selected from the best papers presented in the conference and from invited papers for distinguished participants in the conference. The topics of the papers cover a wide spectrum of topics from the physical to the application 
layers in the communication systems.

The first paper entitled "Tools and Architectural support for Mobile Phones based Crowd Control Systems" by Eiman Kanjo [1]. In this paper the authors proposed a Crowd Control approach that explores the real technical challenges that face the developer of such systems and to move the handling of these volatile properties of pervasive environments to the protocol level. Through the development of two mobile applications, the authors discuss the underlying principles of message passing among crowds and present a set of visual tools that facilitate the adaptation process.

The second paper entitled "Delay Tolerant Routing Protocol Modeling for Low Power Wearable Wireless Sensor Networks" by the authors Muhannad Quwaider and Subir Biswas [2]. The paper presents a structural model for packet store-and-forward routing protocols in Wireless Body Area Networks (WBAN). WBAN has been experimentally constructed in order to illustrate and capture on-body topology disconnections. In this paper, source-to-destination packet routing delay for different protocols like, opportunistic, random-based, utility-based and other protocols that capture multi-scale neighborhoods in human postural mobility has been constructed and evaluated. Then, performance results of the proposed protocols are evaluated experimentally and compared with the results obtained via the model that is developed. Through multi-scale modeling of the on-body spatio temporal neighborhood of the link disconnection patterns, it is shown that the proposed protocols can provide enhanced routing performance compared with a number of existing opportunistic, utility and random based DTN packet routing protocols.

The following paper by Dragana Krstić, Petar Nikolić, and Dragan Radenković is "The Performances of Complex SSC/MRC Combiner in the Presence of Rayleigh Fading" [3]. This paper derived the probability density function (PDF) of dual-branch Switch and Stay/Maximal Ratio Combining (SSC/MRC) combiner output signal at two time instants in the presence of Rayleigh fading. Afterwards, this PDF is used for calculation of the bit error rate (BER), outage probability (Pout) and amount of fading (AF) for complex SSC/MRC combiner for binary phase swift keying (BPSK) modulation, depending on some parameter values. The authors presented the results graphically and point out the gain of using this complex SSC/MRC combiner compared with classical MRC combiners and one channel receiver at one time instant.

The authors Mazen Kharbutli, Monther Aldwairi, and Abdullah Mughrabi contributed the paper entitled "Function and Data Parallelization of Wu-Manber Pattern Matching for Intrusion Detection Systems" [4]. The idea of this paper is to develop a novel and effective parallel implementations of the Wu-Manber (WM) algorithm for signature based detection system. The authors implemented the algorithm and presented and evaluation study for it. The proposed function and data parallel algorithms prove to be effective in terms of execution time reduction and load balancing, thus providing swift intrusion detection at increased network bandwidths. The algorithm achieves an optimal load balance and an average speedup of 2 for four cores.

The last paper named "Fibonacci Backoff Algorithm for IEEE 802.15.4/ZigBee" by the authors Muneer Bani Yassien, Marwa Salayma, Wail Mardini, and Yaser Khamayseh presented a simulation study for the applicability of the Binary Exponential Backoff (BEB) 
function to compute the backoff delay for each node in IEEE 802.15.4 system [5]. Using BEB function, it is possible that two or more nodes may collide if they choose the same backoff exponent value. Consequently this will increase collision and network contention level which will degrade the network overall performance. To overcome this problem, this paper proposes a Fibonacci Backoff (FIB) function to compute the backoff interval. In FIB, each node shall wait for an incremental backoff periods as they need to access the channel. The performance of FIB algorithm is compared against the BEB function.

\section{References}

[1] Eiman Kanjo, "Tools and Architectural support for Mobile Phones based Crowd Control Systems", Network Protocols and Algorithms, Vol. 4, Issue 3, pp. 4 - 14, 2012. http://dx.doi.org/10.5296/npa.v4i3.2052

[2] Muhannad Quwaider and Subir Biswas, "Delay Tolerant Routing Protocol Modeling for Low Power Wearable Wireless Sensor Networks", Network Protocols and Algorithms, Vol. 4, Issue 3, pp. 15 - 34, 2012. http://dx.doi.org/10.5296/npa.v4i3.2054

[3] Dragana Krstić, Petar Nikolić, and Dragan Radenković, "The Performances of Complex SSC/MRC Combiner in the Presence of Rayleigh Fading", Network Protocols and Algorithms, Vol. 4, Issue 3, pp. 35 - 45, 2012. http://dx.doi.org/10.5296/npa.v4i3.2055

[4] Mazen Kharbutli, Monther Aldwairi, and Abdullah Mughrabi, "Function and Data Parallelization of Wu-Manber Pattern Matching for Intrusion Detection Systems", Network Protocols and Algorithms, Vol. 4, Issue 3, pp. 46 - 61, 2012. http://dx.doi.org/10.5296/npa.v4i3.2069

[5] Muneer Bani Yassien, Marwa Salayma, Wail Mardini, and Yaser Khamayseh, "Fibonacci Backoff Algorithm for IEEE 802.15.4/ZigBee", Network Protocols and Algorithms, Vol. 4, Issue 3, pp. 62 - 78, 2012. http://dx.doi.org/10.5296/npa.v4i2.2094

\section{Copyright Disclaimer}

Copyright reserved by the author(s).

This article is an open-access article distributed under the terms and conditions of the Creative Commons Attribution license (http://creativecommons.org/licenses/by/3.0/). 